\title{
Miniplant-Scale Evaluation of a Semibatch-Continuous Tandem Reactor System for the Hydroformylation of Long-Chain Olefins
}

\author{
Michael Jokiel, ${ }^{\dagger}$ Karsten H. G. Rätze, ${ }^{\ddagger}$ Nicolas M. Kaiser, ${ }^{\ddagger}$ Kai U. Künnemann, ${ }^{\S}$ Jan-Peter Hollenbeck, ${ }^{\S}$ \\ Jens M. Dreimann, ${ }^{\S}$ Dieter Vogt, ${ }^{\S}$ and Kai Sundmacher*, ${ }^{*}, \ddagger$

\begin{abstract}
${ }^{\dagger}$ Department Process Systems Engineering, Max Planck Institute for Dynamics of Complex Technical Systems, Sandtorstr. 1, D-39106 Magdeburg, Germany Emil-Figge-Str. 66, D-44227 Dortmund, Germany
\end{abstract} \\ ${ }^{\ddagger}$ Otto-von-Guericke-University Magdeburg, Chair Process Systems Engineering, Universitätsplatz 2, D-39106 Magdeburg, Germany \\ ${ }^{\S}$ Laboratory of Industrial Chemistry, Department of Biochemical and Chemical Engineering, Technical University of Dortmund,
}

Supporting Information

\begin{abstract}
Innovative reactor concepts show evidence to significantly improve the reaction performance in comparison to conventional reactor systems. To evaluate the reactor concepts, experimental investigation of the process behavior is indispensable. In this contribution, a reactor tandem comprising a repeatedly operated semibatch reactor (RSBR) followed by a continuously stirred tank reactor (CSTR) is analyzed for the hydroformylation of 1-dodecene. This reactor tandem was suggested by N. M. Kaiser et al. [Ind. Eng. Chem. Res. 2017, 56, 11507-11518] to increase the selectivity toward the linear aldehyde at high conversion levels of 1dodecene. An additional degree of freedom is gained because of the combined utilization of a batchwise and continuously operated reactor. By using a dynamic process model for planning of the experiments, comparability is ensured with studies of a single CSTR from literature. The experiments confirm an increase in conversion and target product yield applying the RSBR + CSTR tandem, so that up to $90 \%$ selectivity is achieved with closed byproduct recycle.

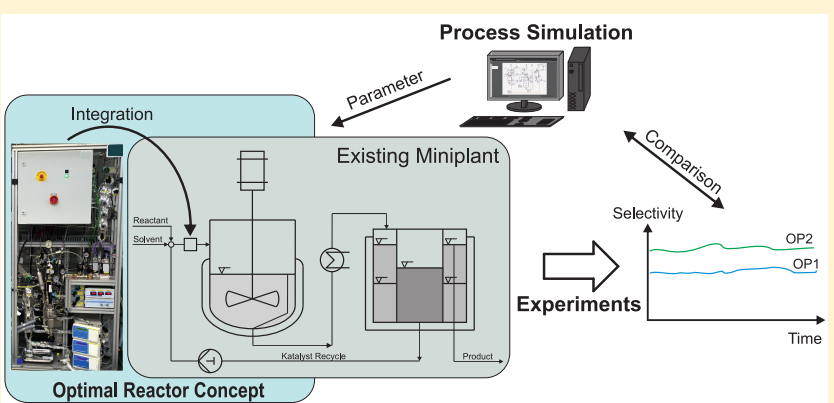

Optimal Reactor Concept
\end{abstract}

\section{INTRODUCTION}

Nowadays, the major part of the produced chemicals are made from petroleum and natural gas, ${ }^{2}$ and in more than $80 \%$ of these processes, catalysts are used. ${ }^{3}$ In the near future, the availability of fossil raw materials is going to decrease. Hence, new production methods and techniques must be investigated for the usage of renewable resources. To achieve this goal, it is required that experts from all involved areas (e.g., chemists, engineers, mathematicians, ect.) join together. With this in mind, the collaborative research center Transregio 63 (CRC/ TR63), Integrated Chemical Processes in liquid multiphase Systems, InPROMPT, has been established in Germany, funded by the German research foundation DFG. The goal of this research center is the development of production processes using homogeneous catalysts and novel multiphase solvent systems. ${ }^{4}$ As model reaction the hydroformylation of 1dodecene was chosen. A special feature of the CRC/TR63 is the demonstration and validation of these processes in miniplants to study their feasibility over a long time period.

For the present study, a repeatedly operated semibatch reactor (RSBR) is integrated into an already existing miniplant that was constructed by Zagajewski et al. ${ }^{5}$ The integration of the RSBR was suggested by Kaiser et al., ${ }^{1}$ in order to maximize the selectivity toward the target product. Two long-term experiments have been performed with the new setup, consisting of the RSBR and a continuously operated stirred tank reactor (CSTR) connected in series. The aim of the first experiment (later denoted as OP1) is the comparison of the performance of this tandem reactor system with the previous results where only a single CSTR was used by Dreimann et al. ${ }^{6}$ In the second experiment (OP2), the behavior of the RSBR + CSTR setup is studied with a closed byproduct recycle. Therefore, the recycle of byproducts from the final distillation column in the original process setup is simulated by adding the corresponding byproducts manually. This allows to realize the integrated overall process while avoiding the operation of the distillation column. The rigorous process model derived by Rätze et al. $^{7}$ is used to determine the flow rates for the experiments as well as the batch reaction times. Finally, all

Special Issue: Reinhard Schomäcker Festschrift

Received: August 14, 2018

Revised: January 4, 2019

Accepted: January 15, 2019

Published: January 15, 2019 
results will be discussed and compared to the literature and the model prediction.

\section{BACKGROUND}

In the following, the reaction network and the applied catalyst recycle technique are described. In addition, experimental results of the original setup are presented and more details on the derivation of the tandem reactor system, used in this work, are given.

2.1. Hydroformylation Reaction. Hydroformylation denotes the reaction between olefins and synthesis gas to synthesize aldehydes. This reaction is widely used to enlarge the chain length of hydrocarbons in order to synthesize alcohols, plasticizers, or solvents. ${ }^{3}$ A challenging task of the hydroformylation is to ensure high chemoselectivities and regioselectivities with respect to the desired aldehyde. ${ }^{8}$ Therefore, transition metals such as rhodium and cobalt are used as catalysts, along with phosphorus-based ligands. ${ }^{8,9}$ For the present study, rhodium is used in combination with a proper ligand leading to an excellent activity and selectivity toward the desired linear aldehyde. ${ }^{3,8}$ The ligand BiPhePhos was chosen by Markert et al. ${ }^{10}$ because it proved to provide the highest selectivity with respect to the linear aldehyde. ${ }^{11,12}$ The reaction network, proposed by Kiedorf et al. ${ }^{13}$ and refined by Hentschel et al., ${ }^{14}$ is shown in Figure 1.

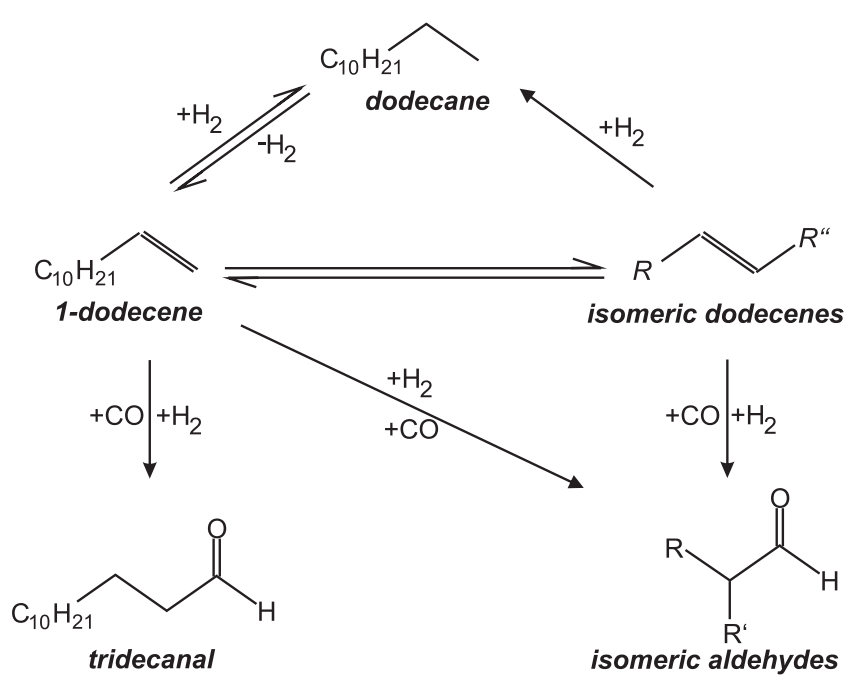

Figure 1. Hydroformylation reaction network $\left(\mathrm{R}=\mathrm{C}_{n} \mathrm{H}_{2 n+1} ; \mathrm{R}^{\prime}=\right.$ $\left.\mathrm{C}_{11-n} \mathrm{H}_{23-2 n} ; \mathrm{R}^{\prime \prime}=\mathrm{C}_{10-n} \mathrm{H}_{21-2 n} ; n=1, \ldots, 5\right)$. [Redesigned figure, adopted with permission from Hentschel et al. ${ }^{14}$ Copyright 2015, American Chemical Society, Washington, DC.]

Besides the main hydroformylation reaction toward the linear and branched aldehdyes, hydrogenation and isomerization reactions occur. The reaction rates of these side reactions are strongly dependent on the synthesis gas composition, as well as the reaction temperature and therefore can be suppressed by applying specific conditions. ${ }^{14,15}$

2.2. Catalyst Recovery. The quantitative recovery of the homogeneous catalyst is the key to enable an economic and sustainable process design. Since the used rhodium and $\mathrm{BiPhePhos}$ ligand are dissolved homogeneously in the reaction mixture, this recovery is a challenging task in the case of high boiling products. Consequently, new recycling techniques, such as aqueous-phase catalysis, aqueous-organic biphasic catalysis, organic-organic biphasic techniques, catalysis using ionic liquids, the usage of supercritical solvents, or soluble polymer-bound catalysts were developed. ${ }^{16,17}$ Since the majority of these solvent systems consist of more than one liquid phase, they are often accompanied by additional masstransfer limitations at the interphases. This led to the development of temperature-controlled multiphase systems.

The idea behind these systems is to perform the chemical reaction in a one phasic system and afterward use a temperature decrease for the separation of the catalyst and the products. ${ }^{18}$ This technique is used in the present study for the catalyst recovery in form of a thermomorphic multiphase system (TMS). The TMS used is composed of three solvents with different polarities: (i) a polar solvent A, (ii) a semipolar solvent $\mathrm{B}$, and (iii) a nonpolar solvent C. ${ }^{18}$ The solvents are to be chosen in a way, such that the polar and nonpolar solvent are not miscible under separation conditions, i.e., decreased temperature. The semipolar solvent $\mathrm{B}$ acts as a temperaturedependent mediator between solvent A and solvent C. ${ }^{19}$ Such a system forms a single phase at higher reaction temperatures and two separate phases at lower temperatures. Depending on the type of reaction, one of these solvents, normally solvent $B$, might even be the reactant itself. ${ }^{19}$ In this work, 1-dodecene is used as substrate for the reaction and as the semipolar solvent for the TMS. Besides 1-dodecene, the TMS consists of $42 \mathrm{wt} \%$ $n$-decane as nonpolar solvent and 42 wt \% N,N-dimethylformamide (DMF) as polar solvent. The rhodium and ligand are dissolved in the polar DMF phase, because of their polarity and hence, can be separated from the reaction mixture at low temperatures. The composition of this system was developed and examined by Schäfer et al. ${ }^{20}$ and Brunsch and Behr. ${ }^{21}$

2.3. Hydroformylation Miniplant. The miniplant consists of a CSTR with a total volume of $1 \mathrm{~L}$, connected to a settler for the phase separation of the TMS. ${ }^{5}$ During the first operation, the requirement of a steady ligand feed was noticed, since branched aldehydes were synthesized in high amounts. Consequently, a separate makeup stream (DMF, rhodium, and ligand) was installed. ${ }^{22}$ With this stream, a conversion of up to $78 \%$ and a tridecanal yield of up to $70 \%$ were achieved, using a molar catalyst:olefin ratio of 1:2000.,22 Later, Dreimann et al. ${ }^{6}$ reported results of another miniplant experiment where only half the catalyst amount used in the reported research of Zagajewski et al. ${ }^{5,22}$ was used. Overall, a tridecanal yield of $63.5 \%$ and a 1-dodecene conversion of $83.5 \%$ to internal dodecenes and linear and branched aldehydes was achieved. These results will be used for the comparison to the outcomes of this study.

In a next step, a distillation column for the separation of the aldehydes from the $n$-decane and byproducts was integrated and operated within the miniplant. ${ }^{23}$ The distillate from the column was fed back into the CSTR to close the second recycle loop of the TMS. Because the recycle stream from the distillation column contained internal olefins, the reaction rate of the 1-dodecene isomerization decreased significantly. A mean tridecanal yield of $54 \%$ and a 1 -dodecene conversion of $67 \%$ were achieved, with a ratio between the linear and branched aldehydes of 95:5, while using a ratio of catalyst:ofelin ratio of 1:4000.

2.4. Optimal Reactor Design. The optimal reactor design by Kaiser et al. ${ }^{1}$ was performed for the hydroformylation process with closed recycles while considering the restrictions of the miniplant setup from Dreimann et al. ${ }^{23}$ The goal for the reactor design was to maximize the selectivity, with respect to the linear aldehyde at high conversions of 1-dodecene $(X \geq$ 


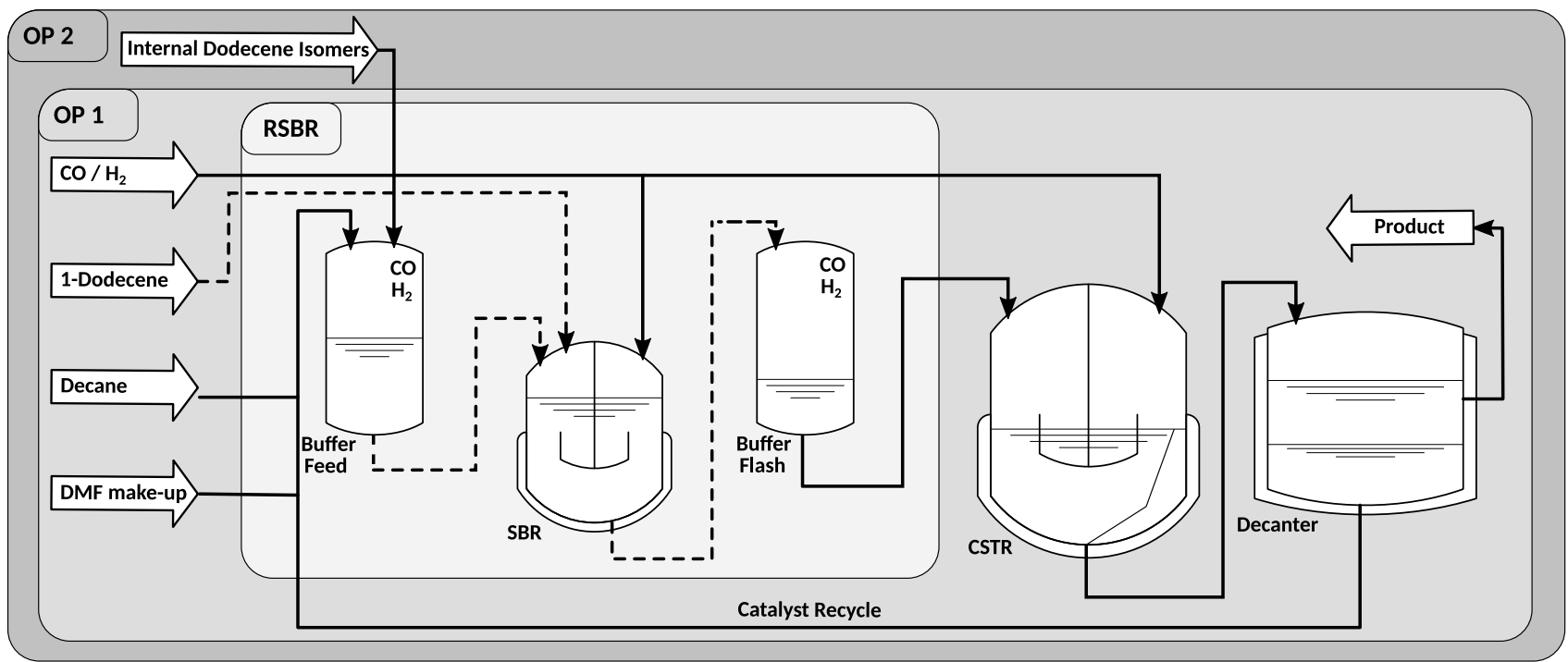

Figure 2. Process flowchart and process units considered in the model for operating points 1 and 2 (dashed lines denote periodical flow; solid lines represent continuous flow).

90). The model-based analysis indicates the beneficial use of a reactor tandem consisting of a distributed sidestream reactor and a back-mixed reactor in series. ${ }^{1}$ The first reactor requires an initial dosing of reactant and distributed dosing of gas, while the second one can be realized with a CSTR. For the technical design of this reactor tandem, two realization possibilities were suggested: (i) a continuous flow reactor followed by a CSTR and (ii) a repeated semibatch reactor followed by a CSTR. An advantage of both realizations is the possibility to use the already-installed CSTR of the miniplant. In addition to the SBR unit, the second realization requires two buffer vessels before and after the reactor. The vessels are periodically charged and discharged during the batch reactions. Furthermore, Kaiser et al. ${ }^{1}$ performed a parameter optimization for both derived tandem reactor systems, which indicates a conversion and selectivity gain of $24 \%$ and $40 \%$ respectively, in comparison to the original miniplant setup. ${ }^{23}$

The RSBR was constructed and first operated with a closed catalyst recycle by Rätze et al. ${ }^{7}$ to demonstrate the experimental feasibility of the reactor concept. However, in contrast to the suggested reactor volume of $550 \mathrm{~mL}$ by Kaiser et al., ${ }^{1}$ the RSBR rig was constructed with a reactor volume of $240 \mathrm{~mL}$. $^{7}$ Rätze et al. ${ }^{7}$ developed a rigorous dynamic process model and validated this dynamic model with the steady-state model used by Kaiser et al. ${ }^{1}$ for the parameter optimization. The developed model of Rätze et al. $^{7}$ is used in this work to perform simulations of the process behavior and to determine the flow rates for the experiments.

\section{PROCESS MODEL}

The repeated operation of a semibatch reactor in a continuous overall process is experimentally challenging due to various degrees of freedom presented to the process operator. Besides different vessel temperatures and pressures, the batch time in each process cycle as well as the volumetric flow rates for all process units need to be controlled. As a consequence, thorough knowledge of the process and experience is required by the operating staff for a successful operation, especially with respect to process startup.
Model-based simulations using time-resolved process models allow for a priori predictions of the dynamic process behavior and may support the experimental investigation. Rätze et al. ${ }^{7}$ introduced a dynamic model of the RSBR process in the TMS miniplant and verified its convergence to the same cyclic steady state predicted by a steady-state process model. Therefore, it is adapted in this contribution and used for planning the experiments and predicting the experimental process behavior.

Based on these predictions, all internal and external volumetric flow rates including the necessary makeup streams can be estimated and used for the experimental operation. Furthermore, the process model is extended to allow for the distribution of the overall process residence time onto each process unit. As a consequence, the batch time for each process cycle can be set, such that a predetermined overall residence time is maintained and comparability between different process setups is ensured.

In the following, the process model variants for both experimental operating points are introduced and the methodological approach for distributing the process residence time is explained. For a detailed description of the different process unit models, the solution procedure of the dynamic model and the initial conditions for the process model, the reader is referred to Rätze et al. ${ }^{7}$

3.1. Process Model Variants. In accordance with the experimental setup, the process model consists of the RSBR, the required feed, and flash buffer vessels, as well as a CSTR and a decanter for liquid-liquid phase separation. In contrast to the process model by Rätze et al., ${ }^{7}$ the distillation column is neglected. For evaluating the performance of the new reactor in the miniplant setup, two operating points that are visualized in Figure 2 are considered.

At the first operating point, the focus lies on the reactor performance without the recycle of internal dodecenes. To compensate for the losses of catalyst, polar and nonpolar solvent, makeup streams are available which ensure the correct solvent to substrate ratio and an adequate liquid holdup in the SBR, in accordance with Dreimann et al. $^{6}$

For the second operating point, the byproduct recycle is considered. In the experimental setup, the nonpolar recycle is 
simulated using a mixture of internal dodecenes (see section 3.2), which is fed alongside the 1-dodecene in each batch. To ensure comparability between the simulations and the experimental investigations, a constant makeup stream for the internal dodecenes is added to the process model (see Figure 2) and determined using

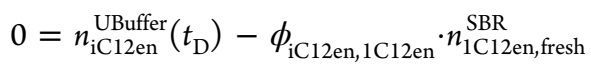

Here, $t_{\mathrm{D}}$ indicates the time of one process cycle, $n_{\mathrm{iC} 12 \mathrm{en}}^{\mathrm{UBuff}}$ represents the molar amount of internal dodecene isomers in

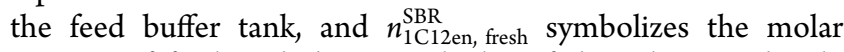
amount of fresh 1-dodecene, which is fed in the next batch. The ratio of internal dodecene isomers and 1-dodecene is represented by

$$
\phi_{\mathrm{iC12en,1C12 \textrm {en }}}=\frac{m_{\mathrm{iC12 \textrm {en }}}^{\mathrm{UBufer}}\left(t_{\mathrm{D}}\right)}{m_{1 \mathrm{C} 12 \mathrm{en}, \mathrm{fresh}}^{\mathrm{SBR}}}
$$

and adjusted in accordance with the desired operating point. Because of the increase in dodecene isomers in the reaction mixture, the solvent and catalyst makeup streams must be adjusted via the equations

$$
\begin{aligned}
& \left(\frac{m_{\mathrm{C} 10 \mathrm{an}}}{m_{\mathrm{DMF}}}\right)^{\text {UBuffer }}\left(t_{\mathrm{D}}\right)=\phi_{\mathrm{C} 10 \mathrm{an}, \mathrm{DMF}} \\
& \left(\frac{m_{\mathrm{C} 10 \mathrm{an}}^{\text {UBuffer }}\left(t_{\mathrm{D}}\right)}{m_{1 \mathrm{C} 12 \mathrm{en}, \mathrm{iC} 12 \mathrm{en}}^{\text {UBuffer }}\left(t_{\mathrm{D}}\right)+m_{1 \mathrm{C} 12 \mathrm{en}, \mathrm{fresh}}^{\mathrm{SBR}}}\right)=\phi_{\mathrm{C} 10 \mathrm{an}, 1 \mathrm{C} 12 \mathrm{en}} \\
& \left(\frac{n_{\mathrm{cat}}\left(t_{\mathrm{D}}\right)}{n_{1 \mathrm{C} 12 \mathrm{en}, \mathrm{iC} 12 \mathrm{en}}}\right)^{\mathrm{UBuffer}}=\phi_{\text {cat, } 1 \mathrm{C} 12 \mathrm{en}}
\end{aligned}
$$

to ensure a substrate:solvent ratio that allows for phase separation in the decanter.

3.2. Experimental Operation Parameters. The operation parameters used in this study are determined semiempirically. Since the comparability to the work of Dreimann et al. $^{6}$ is aspired, the residence time, as well as catalyst and ligand amounts, are chosen to be the same. The reactor temperatures are selected based on former selectivity optimizations of the 1-dodecene hydroformylation network. The studies revealed a temperature increase from $\sim 95-115{ }^{\circ} \mathrm{C}$ within the first $30-40$ min of reaction time. ${ }^{14,15}$ Afterward, a constant temperature is required to increase the backisomerization rate of synthesized internal olefins to 1dodecene, which then can be hydroformylated to tridecanal. Therefore, the temperature in the CSTR is set to $115^{\circ} \mathrm{C}$. For the temperature in the SBR, a constant temperature of $105^{\circ} \mathrm{C}$ is chosen because it is the mean temperature between $95{ }^{\circ} \mathrm{C}$ and $115{ }^{\circ} \mathrm{C}$. Since no adjustment of the $\mathrm{CO}$ and $\mathrm{H}_{2}$ ratio was considered in the work of Dreimann et al., ${ }^{6}$ an equal synthesis gas composition is used in this study as well.

During the second experiment (OP2), two different amounts of internal olefins will be fed, in order to study their influence on the reaction performance. First, a ratio of

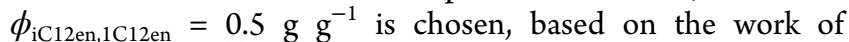
Dreimann et al., ${ }^{23}$ from a former operation of the single CSTR setup with the closed byproduct recycle from the product distillation column. This olefin ratio is denoted as OP2.1, whereas the higher ratio of internal olefins $\left(\phi_{\mathrm{iC} 12 \mathrm{en}, 1 \mathrm{C} 12 \mathrm{en}}=0.75\right.$ $\left.\mathrm{g} \mathrm{g}^{-1}\right)$ is denoted as OP2.2. An overview of all process and simulation parameters can be found in Table S.1 in the Supporting Information.

3.3. Distribution of Residence Time. For the experimental investigation of the RSBR + CSTR setup, a constant residence time of $\tau_{\text {total }}=3.50 \mathrm{~h}$ is chosen, which is in accordance with the data presented by Dreimann et al. ${ }^{6}$ Since the reference setup only consists of a CSTR as a reaction vessel, the residence time $\tau_{\text {total }}$ of the RSBR + CSTR reactor tandem must be distributed onto the SBR, flash buffer vessel, and the CSTR, which are all considered as reaction zones. The feed buffer vessel is not considered as a reaction zone, since the 1-dodecene is directly fed into the SBR. By operating the SBR in cycles, a preparation time for charging and emptying the reactor is necessary. It is generally taken into account in the production cycles of batch processes, ${ }^{24}$ because this additional time is lost for the reaction. Therefore, it reduces the space time yield, in comparison to a fully continuous approach. For the total residence time of the RSBR + CSTR setup, the preparation time is taken into account, so that the distribution of the residence time onto the different reaction zones can be calculated with

$$
\tau_{\text {total }}=t_{\mathrm{B}}+t_{\mathrm{I}}+\tau^{\text {DBuffer }}+\tau^{\mathrm{CSTR}}
$$

where $t_{\mathrm{B}}$ and $t_{\mathrm{I}}$ represent the batch and preparation time, respectively, and $\tau^{\mathrm{DBuffer}}$ and $\tau^{\mathrm{CSTR}}$ abbreviate the residence time in the flash buffer vessel and the CSTR. ${ }^{7}$ The sum of the batch and preparation time,

$$
t_{\mathrm{D}}=t_{\mathrm{B}}+t_{\mathrm{I}}
$$

is denoted as the time of one process cycle to ensure a continuous process operation.

The residence time of an ideally mixed CSTR is defined as

$$
\tau^{\mathrm{CSTR}}=\frac{V_{\mathrm{L}}^{\mathrm{CSTR}}}{\dot{V}_{\text {out }}}
$$

where the volumetric flow rate $\dot{V}_{\text {out }}$ leaving the flash buffer vessel is equal to the volumetric flow rate entering and leaving the CSTR. For the flash buffer vessel, a mean residence time is defined as follows:

$$
\tau^{\text {DBuffer }}=\frac{0.5 \cdot V_{\mathrm{L}}^{\text {DBuffer }}(t=0)}{\dot{V}_{\text {out }}}
$$

By using the dynamic process model $^{7}$ and eqs (6-9) as constraints in the optimization problem, the required batch time to fulfill eq (6) can be calculated for each process cycle.

3.4. Simulation Results. With the extensions for the dynamic process model of Rätze et al. $^{7}$ from section 3, simulations of the process behavior as well as the distribution of the process residence time onto the different process units can be performed. In the following, the calculation results from the distribution of the residence time in steady state are shown for OP1 in Table 1, since neither the flash buffer vessel and

Table 1. Steady-State Residence Times of Different Process Units for Operating Point 1

$\begin{array}{lc}\text { process unit } & \text { steady-state residence time, } \tau / \mathrm{min} \\ \text { SBR } & 28.71 \\ \text { SBR idle } & 30.00 \\ \text { DBuffer } & 31.35 \\ \text { CSTR } & 119.93\end{array}$


CSTR liquid holdup, nor the volumetric flow rate in the continuous process change significantly between both operating points. For better applicability in the experiments, the calculated batch time is rounded to $\tau_{\mathrm{B}} \approx 30 \mathrm{~min}$ and used for the process simulations for operation points 1 and 2 .

All dynamic optimization problems (DOPs) are reformulated in accordance with Rätze et al. ${ }^{7}$ as nonlinear programs (NLPs) using direct collocation on 20 finite elements with 3 collocation points and solved to local optimality using CasADi ${ }^{25}$ and Ipopt 3.12.3 ${ }^{26}$ with the linear solver MA2 $7^{29}$ on a machine with an Intel Core i5-7200U with $8 \mathrm{~GB}$ of RAM. Initialization of the optimization problem is done via integration and a pre optimization step of the SBR to generate a good initial guess for the gas dosing profiles. The process parameters, used in all calculations, can be found in Table S.1 in the Supporting Information. In the following, the simulation results are presented for each operating point.

3.4.1. Operating Point 1. For OP1, the tandem reactor system is operated without the recycle of dodecene isomers (see Figure 2). Figure 3 shows the yield and conversion

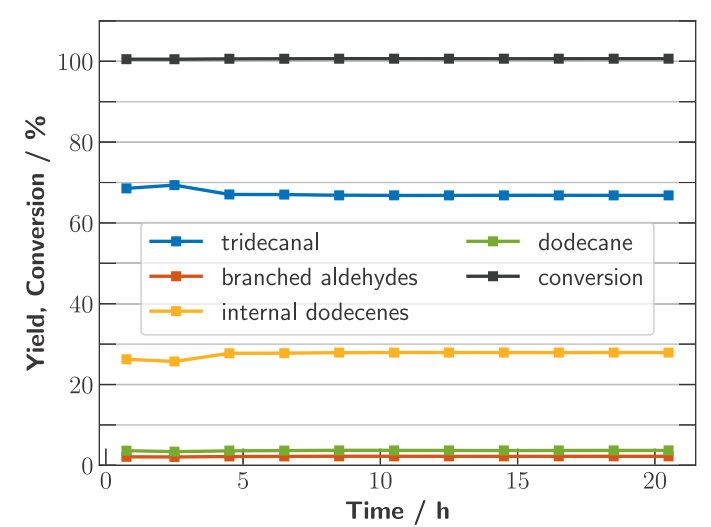

Figure 3. Prediction of the yield and conversion calculated with eqs (S.1) and (S.2) in the Supporting Information of different reaction products for the course of 10 batch cycles for the first operating point.

according to eqs (S.1) and (S.2) in the Supporting Information for 10 process cycles, including the start-up process as well as steady-state operation. After two process cycles, the polar recycle stream is active leading to minor corrections, so that a tridecanal yield of $66.82 \%$ achieved in steady state.

3.4.2. Operating Point 2. The second operating point includes the additional feed of internal dodecenes via eq (1) under consideration of adjusted makeup constraints eqs (3)(5). Here, two different dodecene isomer to 1-dodecene ratios are used, analogous to the experiments (see section 4.3.2) and the conversion as well as the yield calculations are adjusted according to eqs (S.4) and (S.5) (see the Supporting Information) to account for the additional conversion of dodecene isomers. Figures $4 \mathrm{a}$ and $4 \mathrm{~b}$ show the conversion and yield of tridecanal, branched aldehydes, and dodecane over 15 process cycles, including the start-up and steady-state phase for operating point 2.1 and 2.2, respectively. For OP2.1, the product yields show minor changes in the first $8 \mathrm{~h}$ until converging to a constant product distribution. During startup, the tridecanal yield reduces from $62.62 \%$ to $62.16 \%$, which is due to the increase in recycled aldehydes via the polar decanter recycle and the subsequent decrease in hydroformylation activity.

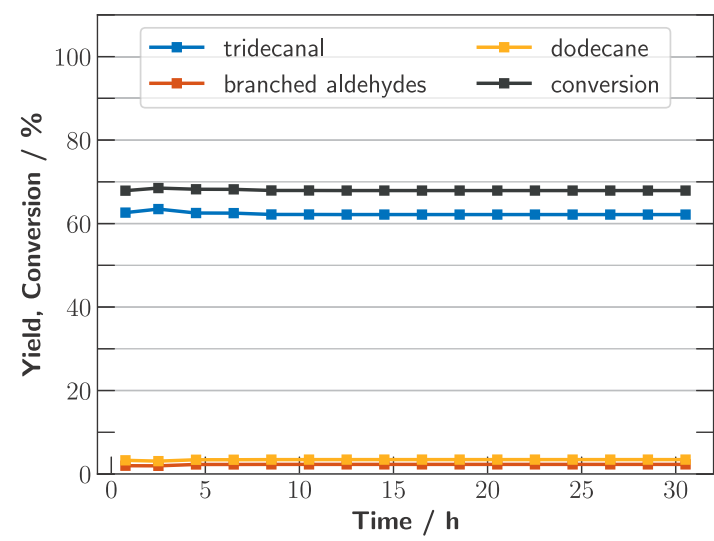

(a) Operating point 2.1

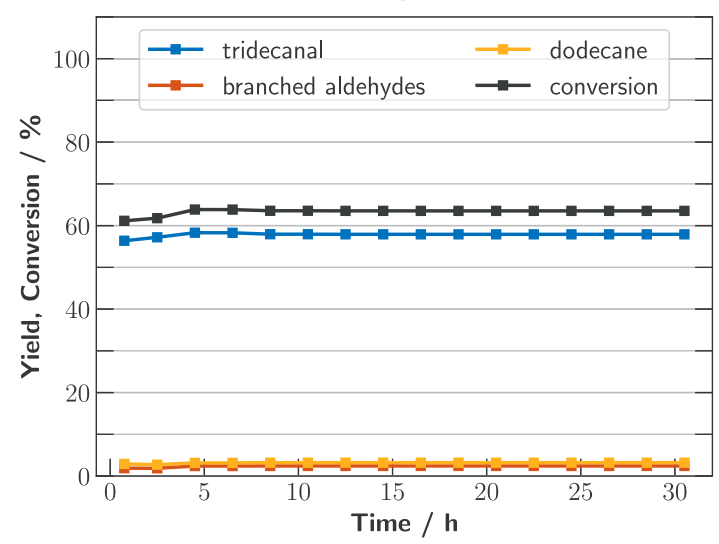

(b) Operating point 2.2

Figure 4. Prediction of the yield and conversion calculated with eqs (S.4) and (S.5) (see the Supporting Information) of different reaction products over the course of 15 process cycles.

For OP2.2, the corrections during startup are more pronounced, since the percentage of dodecene isomers doubled, in comparison to OP2.1. After two process cycles, the polar recycle becomes active, which allows for (by)products to be recycled. As a consequence, a significant amount of tridecanal is recycled, which leads to a lower concentration of dodecene isomers in the reaction mixture. Besides an increased hydroformylation activity, all product yields in Figure $4 \mathrm{~b}$ increase during startup due to the consideration of dodecene isomers as the substrate in eq (S.5) (see the Supporting Information). In the case of tridecanal, the yield increases from $56.37 \%$ to $57.91 \%$.

An overview of the mean yield and selectivity toward the linear aldehyde $S_{\mathrm{nC13al}}$ as well as the mean conversion of olefin $X_{\mathrm{C} 12 \mathrm{en}}$ in steady state can be found in Table 3 in section 5 for the simulations of the different operation points.

\section{MINIPLANT SETUP AND RESULTS}

In the following, the experimental setup and procedure are described. Furthermore, details on the synthesis of the internal olefins are given, and the results from both miniplant experiments are presented.

4.1. Experimental Setup. A simplified flowchart of the process is shown in Figure 2. In addition, Figure 5 depicts a photograph of the miniplant. The RSBR rig is comparable to the one used by Rätze et al. ${ }^{7}$ for the proof of concept. The $n$ decane and the DMF makeup streams are fed continuously with pumps (ProMinent $\mathrm{GmbH}$, Präzisionskolbendosier- 


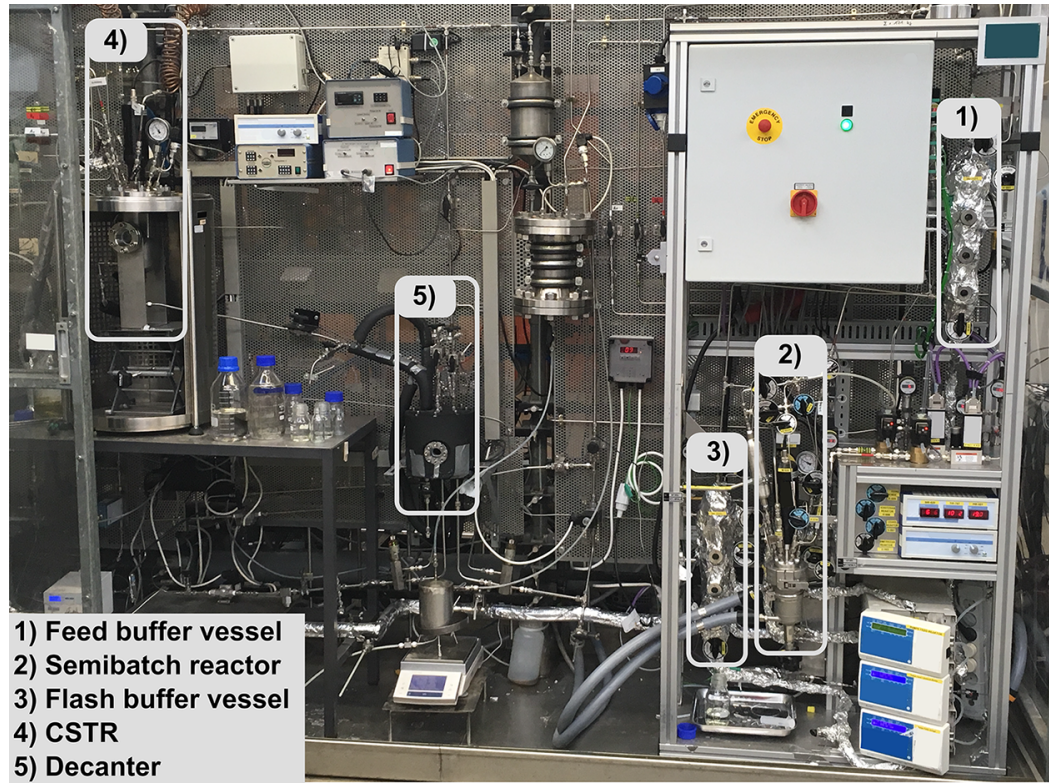

Figure 5. Image of the miniplant with highlighted process units.

pumpe, Model MDLA400600 and Model MDLA4006150) into the feed buffer vessel. For the OP2 experiment, the $n$ decane is substituted by a mixture of $n$-decane and internal olefins. For the start of a new batch, a specified amount from the feed buffer vessel is pumped (KNAUER Wissenschaftliche Geräte GmbH, Model Smartline 1000) into the SBR (Büchi AG, miniclave drive Typ 3, $V=240 \mathrm{~mL}$ ), while 1-dodecene is pumped (KNAUER Wissenschaftliche Geräte $\mathrm{GmbH}$, Model Smartline 1050) into the separate substrate vessel (Swagelok DOT-compliant, $V=150 \mathrm{~mL}$ ). After the preparation time is passed, the substrate vessel is pressurized with synthesis gas by two mass flow controllers (Brooks Instrument, series SLA5800). When the pressure of 24 bar (abs) is reached inside the substrate vessel, the ball valve to the SBR is opened. The pressure difference between both vessels is used to transfer the 1-dodecene into the reactor and the reaction starts immediately. The reaction mixture inside the reactor is stirred continuously with a gassing stirrer $(700 \mathrm{rpm})$.

Temperature and pressure of the SBR are continuously controlled by a thermostat (Julabo GmbH, Model FP40HL) and a pressure controller. This controller is implemented into the process control system and operates the two mass flow controllers. When the reaction time is over, the gas feed into the reactor is stopped. Afterward, the reaction mixture is transferred into the flash buffer vessel by the usage of the pressure difference between the two vessels. This is done 4-6 times by alternatingly pressurizing and depressurizing both vessels. Subsequently, the reaction mixture is continuously pumped (KNAUER Wissenschaftliche Geräte GmbH, Model Smartline 1050) from the flash buffer vessel into the CSTR (Büchi AG, $V=1 \mathrm{~L}$ ). The temperature and the pressure inside the CSTR are controlled by a thermostat (Julabo $\mathrm{GmbH}$, Model 6-ME) and two mass flow controllers (Bronkhorst High-Tech B.V., Model F200 CV). The vessel is stirred with a speed of $800 \mathrm{rpm}$. The liquid holdup inside the CSTR is kept constant by means of an overflow pipe which is directly connected to the decanter (self-built, made from stainless steel, $V_{\text {tot }}=495 \mathrm{~mL}$ ). For pressure compensation, the gas phases in both vessels are connected to each other. The temperature inside the decanter is controlled by a thermostat (Julabo
$\mathrm{GmbH}$, Model F33-ME). The reaction mixture is cooled to 5 ${ }^{\circ} \mathrm{C}$ inside the decanter and hence, splits into two phases. Samples from the product phase are taken and analyzed via gas chromatography (GC) (Agilent Technologies, Model 7890A, HP-5 column, FID) in intervals of $60 \mathrm{~min}$. The catalyst phase is continuously pumped (KNAUER Wissenschaftliche Geräte $\mathrm{GmbH}$, Model S4.1S) back into the feed buffer vessel. For the compensation of the negative pressure difference between the decanter (21 bar (abs)) and the buffer vessel (4-7 bar (abs)), a relief valve (Swagelok R-Series) is installed directly after the pump. The product phase is continuously taken out of the decanter with an overflow pipe and a liquid detector (ACS Control-Systems GmbH, Model Vibrocont SCM) combined with an electromagnetic valve (Bürkert $\mathrm{GmbH} \& \mathrm{Co}$. KG, Model 0255). A separate flash vessel (self-built from stainless steel) is then used to depressurize the product phase.

The electronic devices of the RSBR rig are operated from the process control system (Siemens PCS7, Version 8.1). The other part of the miniplant setup is controlled by a LabVIEW system (National Instruments, Version 2010). All pipes through which the TMS flows and the two buffer vessels are heated by electric heating ducts (Horst $\mathrm{GmbH}$ ) to prevent phase separation of the TMS. Sight glasses (Herberts Industrieglas $\mathrm{GmbH} \& \mathrm{Co} \mathrm{KG}$ ) are integrated into the two buffer vessels (made from Swagelok $1 \mathrm{ft}$ fittings) for a visual observation of the liquid level. The chemicals used in the miniplant experiments are listed in Table 2.

4.2. Isomerization of 1-Dodecene. For the simulated byproduct recycle during the OP2 experiment, internal $\mathrm{C} 12$ olefins are required. Since the isomers are not commercially

Table 2. Chemicals for the Miniplant Experiments

\begin{tabular}{lcc}
\multicolumn{1}{c}{ substance } & manufacturer & purity \\
$n$-decane & VWR & $>95 \%$ \\
DMF & VWR & $>99 \%$ \\
1 -dodecene & Merck & $>94 \%$ \\
BiPhePhos & Molisa & $>99 \%$ \\
$\operatorname{Rh}($ acac $)(\mathrm{CO})_{2}$ & Umicore & $>39 \% \mathrm{Rh}$
\end{tabular}




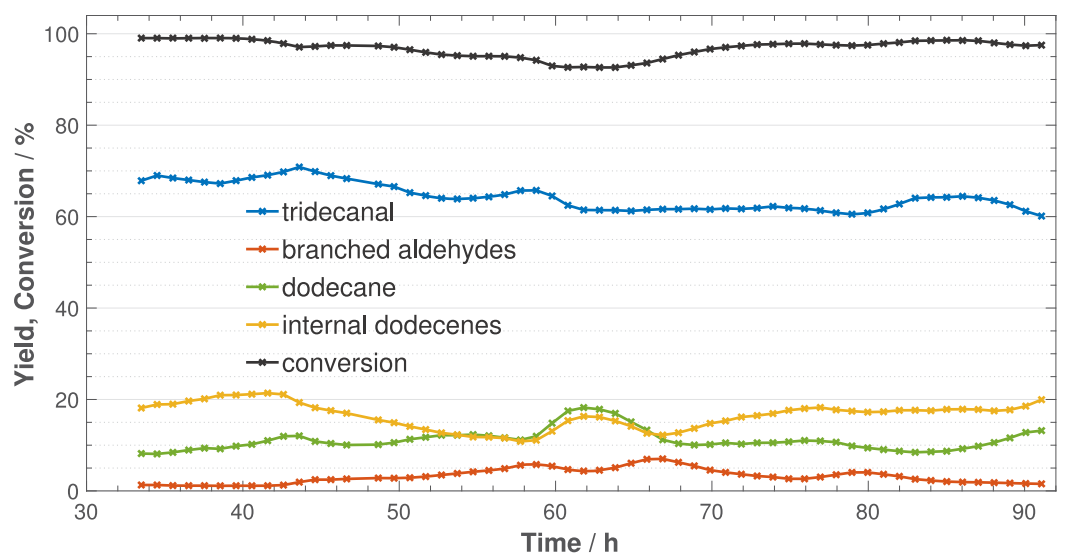

Figure 6. Achieved yields and conversion during the miniplant experiment OP1.

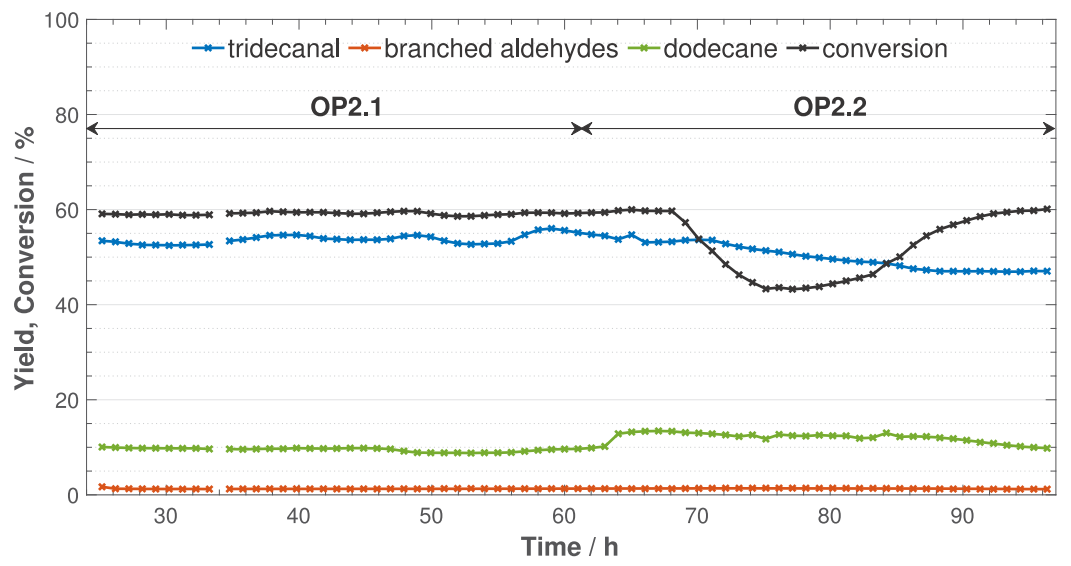

Figure 7. Achieved yields and conversion during the second miniplant experiment OP2.

available, 1-dodecene is isomerized to synthesize a mixture of internal olefins. This is done batchwise in a $1 \mathrm{~L}$ autoclave from Büchi AG (versoclave type 4). The vessel is filled with $348 \mathrm{~g}$ of n-decane (Merck, $>99 \%$ purity), 232 g of DMF (SigmaAldrich, $>99 \%$ purity), 145 g of 1 -dodecene (Alfa Aesar, $>97 \%$ purity), $0.0222 \mathrm{~g}$ of catalyst precursor $\mathrm{Rh}(\mathrm{acac})(\mathrm{CO})_{2}$ (Sigma-Aldrich, $>98 \%$ purity) and $0.2234 \mathrm{~g}$ of BiPhePhos (Molisa, $>99 \%$ purity). The molar ratios of catalyst:substrate and catalyst:ligand are $1: 10000$ and $1: 3.3$, respectively. For activating the catalyst, the reactor is flushed and evacuated five times with synthesis gas under intensive stirring. Afterward, the vessel is pressurized to 9 bar (abs) with the inert gas argon and heated up to $115^{\circ} \mathrm{C}$. When the temperature is reached, liquid samples are taken after 20, 50, and $90 \mathrm{~min}$, respectively. The samples are immediately diluted with 2-propanol (Honeywell Riedel-de Haën, $>99 \%$ purity) to prevent phase separation of the TMS at room temperature. After $120 \mathrm{~min}$, the autoclave is cooled to room temperature and depressurized. The catalyst containing DMF phase is then separated from the $n$-decane/ dodecene phase under an argon atmosphere. All samples are analyzed by GC and GC/MS (Agilent Technologies, Model 9820 with a HP5 column and flame ionization detection (FID) detector; Agilent Technologies, Model 7890B with a HP5 column and FID/TCD detectors). The results showed that the 1 -dodecene was almost completely isomerized after $20 \mathrm{~min}$. As the isomer mixture is fed during the experiment, along with the $n$-decane feed stream, no separation of the olefins and $n$-decane is necessary. Six batch isomerizations were necessary to synthesize enough dodecene isomers for the OP2 experiment.
The dodecene/ $n$-decane mixtures contained $29-30$ wt $\%$ dodecene isomers and $<1$ wt \% DMF.

4.3. Experimental Results. The miniplant was operated continuously during both experiments for more than $90 \mathrm{~h}$. The results are presented in the following.

4.3.1. Results from Experiment OP1. In the first miniplant experiment, 93 batch reactions with the RSBR rig were performed. The catalyst recycle loop was closed $6 \mathrm{~h}$ after the startup. The yields calculated from the online GC data are shown in Figure 6. According to eq (S.1), a mean 1-dodecene conversion of $95.1 \%(\mathrm{SD}=12.5 \%)$ during the entire operation time and a tridecanal yield (according to eq (S.2)) of $63.2 \%$ ( $\mathrm{SD}=8.9 \%)$ were achieved. A dodecane yield of $11 \%$ is visible in Figure 6 during the entire experiment. The yield of the undesired branched aldehydes increased during the middle part of the experiment (see Figure 6). Therefore, the flow rate of the DMF makeup stream was increased by $8 \mathrm{~mL} / \mathrm{min}$ for 6 $\mathrm{h}$ to enhance the ligand concentration in the miniplant. However, this increased the concentration of catalyst, because the stream also contains 0.015 wt \% Rh-precursor. Becuase of the higher BiPhePhos concentration, the ratio between the linear and branched aldehyde increased again, from 90:10 at hour 67 to $97: 3$ until the end of the campaign.

For analyzing the rhodium and phosphorus leaching by ICPOES, samples from the product phase were taken. The average leaching of rhodium and phosphorus was $1.4 \% / \mathrm{h}$ and $1.39 \% / \mathrm{h}$, respectively, based on the initial amount used.

4.3.2. Results from Experiment OP2. The synthesized dodecene isomer/n-decane mixture (see section 4.2 ) is diluted 
Table 3. Comparison of the Experimental and Predicted Dodecene Conversions As Well As Tridecanal Yields and Selectivies ${ }^{a}$

\begin{tabular}{|c|c|c|c|c|c|c|c|}
\hline & \multirow[b]{2}{*}{ Dreimann et al. ${ }^{6}$} & \multicolumn{3}{|c|}{ Experimental } & \multicolumn{3}{|c|}{ Model Prediction } \\
\hline & & OP1 & OP2.1 & OP2.2 & OP1 & OP2.1 & OP2.2 \\
\hline $\mathbf{X}_{\mathrm{C12 \textrm {en }}} / \%$ & 84.5 & $95.1(80.3)$ & $(59.2)$ & $(59.3)$ & $99.5(70.7)$ & $99.4(66.7)$ & $99.3(62.3)$ \\
\hline $\mathbf{Y}_{\mathrm{nC13al}} / \%$ & 62.5 & $63.2(64.4)$ & (53.4) & $(47.0)$ & $66.8(66.0)$ & $78.0(62.2)$ & $87.1(57.9)$ \\
\hline $\mathrm{S}_{\mathrm{nC13al}} / \%$ & 74 & $66.5(80.2)$ & $(90.2)$ & $(79.3)$ & $67.1(93.3)$ & $78.5(93.2)$ & $87.8(93.0)$ \\
\hline
\end{tabular}

${ }^{a_{T}}$ The numbers without brackets were calculated with eqs (S.1) -(S.3) in the Supporting Information; numbers shown with brackets were calculated with eqs (S.4) -(S.6) in the Supporting Information.

with $n$-decane until the specified amount of internal olefins is reached. This mixture is fed into the miniplant instead of the $n$ decane. To evaluate the data of this experiment, one must consider that the additionally fed isomers are a potential educt for all chemical reactions (see Figure 1). Since it is not possible to differentiate between the fed dodecene isomers and the synthesized ones in the miniplant, the sum of the fed 1dodecene and internal olefins is considered as educt for the evaluation of the conversion eq (S.4) (see the Supporting Information), as well as the yield eq (S.5) (see the Supporting Information) and selectivity eq (S.6) (see the Supporting Information). The achieved yields of the second miniplant experiment with 100 completed batch reactions are shown in Figure 7. Compared to the first experiment, less fluctuations are observable, indicating a more-stable operation than in the previous experiment. After $60 \mathrm{~h}$, the dodecene isomer to 1dodecene ratio was enhanced to $0.75 \mathrm{~g} / \mathrm{g}^{-1}$. The miniplant then needed $\sim 25 \mathrm{~h}(7.1$ times the residence time) to stabilize again (Figure 7). This period indicates that the composition inside the decanter and therefore the catalyst recycle stream were also influenced by the feed ratio of dodecene isomers. Equation (S.4) reveals, with the lower dodecene isomer amount, a conversion of $59.2 \%(\mathrm{SD}=0.3 \%)$ and eq (S.5) reveals a tridecanal yield of $53.4 \%(\mathrm{SD}=1.0 \%)$. With the conditions of OP2.2, a conversion of $59.3 \%(\mathrm{SD}=7.9 \%)$ and a tridecanal yield of $47.0 \%$ ( $S D=0.1 \%$ ) were achieved during the last $7 \mathrm{~h}$. The small standard deviations confirm the more stable operation of the miniplant during this experiment. The ratio of the linear to branched aldehydes is always $>97: 3$ and hence higher than that observed in the first experiment. Again, a large mean yield (OP2.1: 6.6\% and OP2.2: 6.6\%) of the hydrogenation product can be observed in Figure 7. The results of the ICP-OES analysis show an average leaching of $0.74 \% / \mathrm{h}$ for rhodium and $1.35 \% / \mathrm{h}$ for phosphorus based on the initial amount used. These values were stable over the entire time of operation.

\section{DISCUSSION}

Finally, the results of the miniplant experiments are compared to the results published by Dreimann et al. ${ }^{6}$ and the predictions of the model. For this purpose, the tridecanal selectivities and yields, together with the dodecene conversions, are summarized in Table 3. A comparison of the OP1 experiment to the results of Dreimann et al. ${ }^{6}$ exhibits a higher conversion and yield but a lower tridecanal selectivity, because more byproducts, especially dodecane, were synthesized. The reaction time used in this study was 30 min shorter than the reaction time used by Dreimann et al. ${ }^{6}$ as the preparation time of the SBR is considered in the total residence time as well. Hence, the total reaction time was not equal to the total reaction time of Dreimann et al. $^{6}$ This time difference was finally missing to gain more tridecanal selectivity.
A comparison of OP1 and OP2.1 selectivities reveals an increase of $\sim 10 \%$. This gain in selectivity is the result of the internal olefin feed, which lowered the reaction rate of the dodecene isomerization. However, as the dodecene isomer feed rate of OP2.2 was applied, the selectivity decreased down to the level of OP1, but the conversion remained the same. The outcome of the OP2 experiment cannot be directly compared to the work of Dreimann et al., ${ }^{23}$ who actually fed the distillate stream back from the column, because different reaction times were applied.

The experimental tridecanal selectivity of OP1 and the prediction of the model are in excellent agreement, although a yield difference of up to $4 \%$ occurs. This difference is slightly bigger than the error ranges of the model and the experimental data. The selectivity and conversion predictions for OP2.1 show a distinction of $\sim 3 \%$, which is still very good. However, for OP2.2, the model predictions show no decrease of tridecenal selectivity as the experimental data. Therefore, it is likely that a semistable operation point of the miniplant occurred because the feed amount of internal olefins was changed during the operation. Nevertheless, the comparison proofed the ability of the process model to predict the performance of the miniplant.

Compared to the proposed selectivity and conversion gain of the RSBR + CSTR reactor tandem by Kaiser et al., ${ }^{1}$ the experimentally determined increase is lower. The main reasons for this discrepancy are the lower residence time and the different reaction conditions used in the experiments. Kaiser et al. ${ }^{1}$ applied a total residence time of up to $300 \mathrm{~min}$ and dynamic control variables for the SBR whereby a higher tridecanal selectivity results. Nevertheless, the experimental results confirm the ability of the tandem reactor system to enhance the conversion of 1-dodecene and the tridecanal yield.

In terms of flexibility, the RSBR + CSTR tandem must be preferred over the former single CSTR setup. The batch reaction time is an additional time horizon through which the reaction parameters can be changed in order to maximize the process performance. In this study, the entire potential of the RSBR + CSTR setup was not exploited, as the reaction parameters of the RSBR were not changed in time and semiempirical parameters were used. The results of this study serve as a solid basis for further experimental investigations with this setup, including other types of homogeneously catalyzed gas-liquid reactions or even tandem reactions like the hydroaminomethylation. Moreover, the reactor setup is potentially suitable for shorter-chain olefins such as decene. Considering the same TMS as well as catalyst and ligand, the optimal reaction control profiles for decene ${ }^{28}$ are similar to those of dodecene. ${ }^{14,15}$

Finally, some remarks on the operation effort of this reactor network are given. Compared to the single CSTR setup previously discussed, the effort to operate the tandem reactor system is significantly higher. Especially because of the fact that 
many steps of the RSBR operation procedure must be performed manually, like the charging and discharging of the reactor. However, many steps of this procedure can be implemented into a routine and executed by the process control system. The routine is then repeated for every batch, whereby the operation effort will be reduced significantly. This furthermore has the advantage that no additional investment capital is necessary.

\section{SUMMARY}

A tandem reactor system consisting of a repeated semibatch reactor (RSBR) and a continuously stirred tank reactor (CSTR) for the hydroformylation of 1-dodecene was operated in two long-term experiments with closed catalyst recycle loop. Kaiser et al. ${ }^{1}$ suggested this reactor tandem to maximize the selectivity of the linear aldehyde for high conversions of the terminal olefin within a process with closed byproduct recycle loop. The experiments were designed to be comparable in terms of the residence time as well as catalyst and ligand concentrations to Dreimann et al., ${ }^{6}$ who used a single CSTR. The dynamic model from Rätze et al. ${ }^{7}$ was used to distribute the fixed residence time between the process units and to simulate the process beforehand, whereby the flow rates for the experiments as well as the process performance were predicted. The results of the first experiment show a higher conversion and tridecanal yield compared to the single CSTR setup. ${ }^{6}$ During the second experiment, internal olefins were fed additionally, to simulate the byproduct recycle from the product distillation column. With this, a selectivity of up to $90 \%$ was achieved. However, the increase of the internal olefin feed ratio during the experiment decreased the tridecanal selectivity to $79 \%$. The selectivities of the first and the second experiment, where a low internal olefin ratio was fed, are excellently predicted from the process model. The outcome of this study proves the feasibility of the model-based approach from Kaiser et al. ${ }^{1}$ and the capability of the RSBR + CSTR tandem reactor system to enhance the selectivity to the linear aldehyde with closed byproduct recycle loop. Furthermore, the degree of freedom of the used setup is far greater than that of the former single CSTR, which helps to enhance the economy of the process even more.

\section{ASSOCIATED CONTENT}

\section{S Supporting Information}

The Supporting Information is available free of charge on the ACS Publications website at DOI: 10.1021/acs.iecr.8b03874.

Reactor indices; simulation parameters; experimentally determined conversion and yields; associated references (PDF)

\section{AUTHOR INFORMATION}

\section{Corresponding Author}

E-mail: sundmacher@mpi-magdeburg.mpg.de.

\section{ORCID $\odot$}

Michael Jokiel: 0000-0003-0080-081X

Kai Sundmacher: 0000-0003-3251-0593

\section{Notes}

The authors declare no competing financial interest.

\section{ACKNOWLEDGMENTS}

This work is part of the Collaborative Research Center/ Transregio 63, "Integrated Chemical Processes in Liquid Multiphase Systems" (subproject B1, B5). Financial support by the Deutsche Forschungsgemeinschaft (DFG, German Research Foundation) is gratefully acknowledged (SFB/TR63). The author Karsten H. G. Rätze is also affiliated to the "International Max Planck Research School (IMPRS) for Advanced Methods in Process and Systems Engineering (Magdeburg)". Furthermore, the authors like to thank Markus Ikert for his support during the experiments and Umicore AG $\&$ Co. $\mathrm{KG}$ for the donation of the rhodium precursor $\mathrm{Rh}(\mathrm{acac})$ $(\mathrm{CO})_{2}$.

\section{NOMENCLATURE}

\section{Abbreviations}

acac $=$ acetylacetonato

BiPhePhos = 6,6' $\left[\left(3,3^{\prime}\right.\right.$-ditert-butyl-1-5,5' -dimehtoxy-1,1' biphenyl-2,2' diyl) bis(oxy)]bis(dibenzo[d,f][1,3,2] dioxaphosphepin

$\mathrm{CO}=$ carbon monoxide

CSTR = continuously stirred tank reactor

$\mathrm{nC} 12 \mathrm{an}=$ dodecane

$1 \mathrm{C} 12 \mathrm{en}=1$-dodecene

$\mathrm{iC} 12 \mathrm{en}=$ dodecene isomers

C10an $=n$-decane

DMF $=N, N$-dimethylformamide

DOP = Dynamic Optimization Problem

$\mathrm{GC}=$ gas chromatography

$\mathrm{H}_{2}=$ hydrogen

$\mathrm{iC} 13 \mathrm{al}=$ isomeric aldehydes

ICP-OES = inductively coupled plasma-optical emission spectroscopy

IPOPT = Interior Point Optimizer (software name)

$\mathrm{NLP}=$ nonlinear program

$\mathrm{ODE}=$ ordinary differential equation

$\mathrm{Rh}=$ rhodium

$\mathrm{RSBR}=$ repeated semibatch reactor

$\mathrm{SBR}=$ semibatch reactor

$\mathrm{SD}=$ standard deviation

$\mathrm{nC} 13 \mathrm{al}=$ tridecanal

TMS = thermomorphic multiphase system

tot $=$ total

\section{Greek Symbols}

$$
\begin{aligned}
& \varepsilon=\text { hold-up }\left[\mathrm{mL} \mathrm{mL}^{-1}\right] \\
& \tau=\text { residence time }[\mathrm{s}] \\
& \phi=\text { mass or molar ratio }[\mathrm{g} / \mathrm{g}, \mathrm{mol} / \mathrm{mol}]
\end{aligned}
$$

\section{Latin Symbols}

$k_{\mathrm{L}} a=$ volumetric mass-transfer coefficient $\left[\mathrm{min}^{-1}\right]$

$m=$ mass $[\mathrm{kg}]$

$\dot{N}=$ molar flow $\left[\mathrm{mol} \mathrm{s}^{-1}\right]$

$n=$ amount of moles [mol]

$p=$ pressure $[$ bar $(\mathrm{abs})]$

$S=$ selectivity $\left[\mathrm{mol} \mathrm{mol}^{-1}\right]$

$T=$ temperature $[\mathrm{K}]$

$t=$ time $[\mathrm{s}]$

$V=$ volume $[\mathrm{mL}]$

$\dot{V}=$ volumetric flow rate $\left[\mathrm{mL} \mathrm{min}^{-1}\right]$

$X=$ conversion $\left[\mathrm{mol} \mathrm{mol}^{-1}\right]$

$Y=$ yield $\left[\mathrm{mol} \mathrm{mol}^{-1}\right]$

$y=$ gas phase molar fraction $\left[\mathrm{mol} \mathrm{mol}^{-1}\right]$ 


\section{Subscripts and Superscripts}

$\alpha=$ continuous index for species

$\mathrm{AP}=$ apolar

$\mathrm{B}=$ batch

cat $=$ catalyst

DBuffer $=$ flash buffer vessel

Dec $=$ decanter

Dist $=$ distillation

fresh $=$ fresh substrate entering the process

in $=$ inlet

$\mathrm{I}=$ idle

$\mathrm{L}=$ liquid

OP 1 = operating point 1

$\mathrm{OP} 2=$ operating point 2

out $=$ outlet

tot $=$ total

UBuffer $=$ feed buffer vessel

\section{REFERENCES}

(1) Kaiser, N. M.; Jokiel, M.; McBride, K.; Flassig, R. J.; Sundmacher, K. Optimal reactor design via flux profile analysis for an integrated hydroformylation process. Ind. Eng. Chem. Res. 2017, 56, 11507-11518.

(2) Green, M.; Wittcoff, H. A. Organic Chemistry Principles and Industrial Practice; Wiley-VCH Verlag $\mathrm{GmbH} \&$ Co. KGaA: Weinheim, Germany, 2003.

(3) Behr, A. Angewandte Homogene Katalyse; Wiley-VCH Verlag GmbH \& Co. KGaA: Weinheim, Germany, 2008.

(4) InPrompt, https://www.inprompt.tu-berlin.de/index.php?id=17; accessed Jan. 31, 2018.

(5) Zagajewski, M.; Behr, A.; Sasse, P.; Wittmann, J. Continiously operated miniplant for the rhodium catalyzed hydroformylation of 1dodecene in a thermomorphic multi- component solvent system (TMS). Chem. Eng. Sci. 2014, 115, 88-94.

(6) Dreimann, J.; Lutze, P.; Zagajewski, M.; Behr, A.; Górak, A.; Vorholt, A. J. Highly integrated reactor-separator systems for the recycling of homogeneous catalysts. Chem. Eng. Process. 2016, 99, 124-131.

(7) Rätze, K. H. G.; Jokiel, M.; Kaiser, N. M.; Sundmacher, K. Cyclic Operation of a Semi-Batch Reactor for the Hydroformylation of Long-Chain Olefins and Integration in a Continuous Production Process. Chem. Eng. J. 2018, DOI: 10.1016/j.cej.2018.11.151.

(8) Franke, R.; Selent, D.; Börner, A. Applied Hydroformylation. Chem. Rev. 2012, 112, 5675-5732.

(9) Börner, A.; Franke, R. Hydroformylation: Fundamentals, Processes, and Applications in Organic Synthesis; Wiley-VCH Verlag GmbH \& Co. KGaA: Weinheim, Germany, 2016.

(10) Markert, J.; Brunsch, Y.; Munkelt, T.; Kiedorf, G.; Behr, A.; Hamel, C.; Seidel-Morgenstern, A. Analysis of the reaction network for the Rh-catalyzed hydroformylation of 1-dodecene in a thermomorphic multicomponent solvent system. Appl. Catal., A 2013, 462-463, 287-295.

(11) Packett, D. L.. Hydroformylation process for producing 1,6hexanedials. U.S. Patent5,312,996, 1994.

(12) Paciello, P.; Siggel, L.; Kneuper, H.-J.; Walker, N.; Roper, M. Structure-activity relationship for chelating phosphite lig- ands used in rhodium-catalyzed hydroformylations. J. Mol. Catal. A: Chem. 1999, $143,85-97$.

(13) Kiedorf, G.; Hoang, D. M.; Müller, A.; Jörke, A.; Markert, J.; Arellano-Garcia, H.; Seidel-Morgenstern, A.; Hamel, C. Kinetics of 1dodecene hydroformylation in a thermomorphic solvent system using a rhodium-biphephos catalyst. Chem. Eng. Sci. 2014, 115, 31-48.

(14) Hentschel, B.; Kiedorf, G.; Gerlach, M.; Hamel, C.; SeidelMorgenstern, A.; Fre-und, H.; Sundmacher, K. Model-based identification and experimental validation of the optimal reaction route for the hydroformylation of 1-dodecene. Ind. Eng. Chem. Res. 2015, 54, 1755-1765.
(15) Kaiser, N. M.; Flassig, R. J.; Sundmacher, K. Probabilistic reactor design in the framework of elementary process function. Comput. Chem. Eng. 2016, 94, 45-59.

(16) Cornils, B., Hermann, W., Horváth, I., Leitner, W., Mecking, S., Olivier- Bourbigo, H., Vogt, D., Eds. Multiphase Homogeneous Catalysis; Wiley-VCH Verlag GmbH \& Co. KGaA: Weinheim, Germany, 2005.

(17) Kokkinos, N. C.; Kazou, E.; Lazaridou, A.; Papadopoulos, C. E.; Psaroudakis, N.; Mertis, K.; Nikolaou, N. A potential refinery process of light-light naphtha olefins conversion to valuable oxygenated products in aqueous media-Part 1: Biphasic hydroformylation. Fuel 2013, 104, 275-283.

(18) Behr, A.; Fängewisch, C. Temperature- dependent multicomponent solvent systems - an alternative concept for recycling homogeneous catalysts. Chem. Eng. Technol. 2002, 25, 143-147.

(19) Behr, A.; Henze, G.; Johnen, L.; Awun- gacha, C. Advances in thermomorphic liquid/liquid recycling of homogeneous transition metal catalysts. J. Mol. Catal. A: Chem. 2008, 285, 20-28.

(20) Schäfer, E.; Brunsch, Y.; Sadowski, G.; Behr, A. Hydroformylation of 1-dodecene in the thermomorphic solvent system dimethylformamide/decane. Phase behavior-reaction performancecatalyst recycling. Ind. Eng. Chem. Res. 2012, 51, 10296-10306.

(21) Brunsch, Y.; Behr, A. Temperature- Controlled Catalyst Recycling in Homogeneous Transition-Metal Catalysis: Minimization of Catalyst Leaching. Angew. Chem., Int. Ed. 2013, 52, 1586-1589.

(22) Zagajewski, M.; Dreimann, J.; Behr, A. Verfahrensentwicklung vom labor zur miniplant: Hydroformylierung von 1-Dodecen in termomorphen Lösungsmit- telsystemen. Chem. Ing. Tech. 2014, 86, 449-457.

(23) Dreimann, J. M.; Warmeling, H.; Weimann, J. N.; Künnemann, K.; Behr, A.; Vorholt, A. J. Increasing selectivity of the hydroformylation in a miniplant: catalyst, solvent and olefin recycle in two loops. AIChE J. 2016, 62, 4377-4383.

(24) Müller-Erlwein, E. Chemische Reaktionstechnik, 3rd Edition; Springer: Fachmedien Wiesbaden, 2015.

(25) Andersson, J. A General-Purpose Software Framework for Dynamic Optimization. Ph.D. Thesis, Arenberg Doctoral School, KU Leuven, Department of Electrical Engineering (ESAT/SCD) and Optimization in Engineering Center, Heverlee, Belgium, 2013.

(26) Wächter, A.; Biegler, L. T. On the implementationof an interior-point filter linesearch algorithm for large-scale nonlinear programming. Math. Program. 2006, 106, 25-57.

(27) HSL. A collection of Fortran codes for large scale scientific computation. Available via the Internet at: http://www.hsl.rl.ac.uk/.

(28) Jörke, A.; Gaide, T.; Behr, A.; Vorholt, A.; Seidel-Morgenstern, A.; Hamel, C. Hydroformylation and tandem isomerization-hydroformylation of $\mathrm{n}$-decenes using a rhodium-BiPhePhos catalyst: Kinetic modeling, reaction network analysis and optimal reaction control. Chem. Eng. J. 2017, 313, 382-397. 